\title{
Evaluation of the Efficacy of a Small CdTe $\gamma$-Camera for Sentinel Lymph Node Biopsy
}

\author{
Makoto Tsuchimochi ${ }^{1}$, Kazuhide Hayama ${ }^{1}$, Takaaki Oda ${ }^{1}$, Masatoshi Togashi ${ }^{1}$, and Harumi Sakahara ${ }^{2}$ \\ ${ }^{I}$ Department of Oral and Maxillofacial Radiology, The Nippon Dental University School of Life Dentistry at Niigata, Niigata, Japan; and \\ ${ }^{2}$ Department of Radiology, Hamamatsu University School of Medicine, Hamamatsu, Japan
}

\begin{abstract}
We previously reported the basic performance of a prototype small cadmium telluride (CdTe) $\gamma$-camera (SSGC) intended for use in radioguided surgeries. In this study, we sought to confirm the favorable previous results and to extend the preliminary findings to examine the efficacy of the SSGC in an animal study and a clinical setting for sentinel lymph node biopsy. Methods: The prototype SSGC (1,024 pixels; field of view, $44.8 \times 44.8 \mathrm{~mm})$, equipped with a parallel-hole collimator, was used in both animal and clinical studies. ${ }^{99 m T c}$-phytate (18.5 MBq) was injected into the tongues and legs of 6 rabbits. In the clinical study, $74 \mathrm{MBq}$ of 99mTc-phytate was injected into peritumoral regions in 8 patients with oral cancer. The detection of hot nodes by the SSGC was compared with that by a conventional scintillation $\gamma$-camera (CGC). Results: The SSGC detected 29 hot nodes in images of 6 rabbits after injection. The number of hot nodes was the same as the number seen in CGC studies, but the CGC required a longer acquisition time to produce comparable images. There were no differences between the SSGC and the CGC in terms of activity ratios and hot node-to-background ratios. The biodistribution of ${ }^{99 m}$ Tc-phytate in removed tissues was evaluated by contact radiography, and radioactivity was assayed with a $\gamma$-well counter. The mean \pm SD radioactivity in specimens was $0.15 \% \pm 0.15 \%$, with a range of $0.01 \%-0.62 \%$. In the clinical study, the SSGC detected 30 hot nodes with a 5- to 60-s acquisition time at $4 \mathrm{~h}$ after injection. The SSGC documented all hot nodes depicted by the CGC at $4 \mathrm{~h}$ after injection. Conclusion: The SSGC showed significant potential for the detection of sentinel lymph nodes in lymphoscintigraphy. The results of the studies suggested that the SSGC facilitates the exploration of hot nodes in sentinel lymph node biopsy.
\end{abstract}

Key Words: lymphoscintigraphy; oncology; semiconductor $\gamma$-camera; CdTe; handheld $\gamma$-camera; sentinel lymph node biopsy; radioguided surgery; oral cancer

J Nucl Med 2008; 49:956-962

DOI: 10.2967/jnumed.108.050740

\section{$\mathbf{R}$} adioguided surgery has been accomplished with the use of $\gamma$-probes, primarily for sentinel lymph node biopsy.

\footnotetext{
Received Jan. 16, 2008; revision accepted Feb. 26, 2008.

For correspondence or reprints contact: Makoto Tsuchimochi, Department of Oral and Maxillofacial Radiology, The Nippon Dental University School of Life Dentistry at Niigata, 1-8 Hamaura-cho, Chuo-ku, Niigata, Niigata 9518580, Japan.

E-mail: tsuchimochi@ngt.ndu.ac.jp

COPYRIGHT @ 2008 by the Society of Nuclear Medicine, Inc.
}

However, $\gamma$-probes can provide only radioactivity counts and variable-pitch audio output based on the intensity of the detected radioactivity. Allowing the surgeon to see the spatial distribution of the increased radioactivity could result in more accurate delineation of the surgical field, thus facilitating the surgery. Therefore, we developed a prototype small semiconductor $\gamma$-camera (SSGC) that provides images of radioactivities for surgical use (1).

For radioguided surgery, the $\gamma$-camera should be miniaturized or handheld to facilitate its application in the restricted space of a surgical field. A semiconductor detector containing cadmium telluride (CdTe) or cadmium zinc telluride $(\mathrm{CdZnTe})$ has shown excellent performance and quality for imaging (2-7). The semiconductor detector is considered to be the most suitable for miniaturization because it does not require the relatively massive photomultiplier tube that characterizes a traditional scintillation/ photomultiplier tube detector. The solid-state technology allows for the fabrication of a camera head that is smaller, lighter, and more portable. CdTe or CdZnTe appears to be the technology of choice for developing an intraoperative imaging system.

The CdTe detector that we chose for the production of our SSGC resulted in better basic performance, including energy resolution and spatial resolution, than that achieved with a conventional scintillation $\gamma$-camera (CGC). The preliminary results of a simulation study for sentinel lymph node biopsy with the SSGC suggested that this camera would identify sentinel node radioactivity during the procedure (1).

Several reports of handheld small $\gamma$-cameras have been published $(1,8-15)$, but studies to date have not verified the effectiveness of small $\gamma$-cameras in depicting nodes with increased radioactivities in the clinical setting of sentinel lymph node biopsy. In this study, we aimed to confirm our earlier results regarding the basic performance of the SSGC and to extend the preliminary findings by using the camera in an animal study and a clinical setting.

\section{MATERIALS AND METHODS \\ SSGC}

The camera head consists of a "pixelized" CdTe module $(32 \times$ 32 individual elements, total of 1,024 pixels) attached to a 
collimator (Acrorad Co. Ltd.) (1). The field of view is $44.8 \times 44.8$ $\mathrm{mm}$. The camera head measures $152 \times 166 \times 65 \mathrm{~mm}$. The collimator consists of a stack of 100 pieces of $0.1-\mathrm{mm}$-thick tungsten mesh sheets, each having square openings measuring 1.2 $\times 1.2 \mathrm{~mm}$ and a space of $0.2 \mathrm{~mm}$ to fit the geometry of CdTe pixels. The camera head weight is $2.7 \mathrm{~kg}$, and the head is attached to the holding arm of the rack (Fig. 1) (1).

The energy resolution (full width at half maximum) for a single element at the center of the field of view is $4.2 \%$ at $141 \mathrm{keV}$ $\left({ }^{99 \mathrm{~m}} \mathrm{Tc}\right)$, and the mean energy resolution of the CdTe detector arrays is approximately $7.8 \%$. The spatial resolution, represented by the full width at half maximum, is $1.56 \pm 0.05$ (mean \pm SD) $\mathrm{mm}$. The detection efficiency is 0.30 count per second per kilobecquerel $\left({ }^{99 \mathrm{~m}} \mathrm{Tc}\right)(1)$.

\section{CGC}

A CGC (SNC-5100R; matrix size, $512 \times 512$ pixels; field of view, $51 \times 38 \mathrm{~cm}$; Shimadzu) equipped with an $\mathrm{NaI}(\mathrm{Tl})$ crystal detector was used for comparison of CGC- and SSGC-generated images. The system resolution at the ${ }^{99 \mathrm{~m}} \mathrm{Tc}$ energy $(141 \mathrm{keV})$ was $10.4 \%$, and the spatial resolution for ${ }^{99 \mathrm{~m}} \mathrm{Tc}$ was $3.5 \mathrm{~mm}$. A parallel-hole low-energy (high-resolution) collimator was used for imaging.

\section{Animal Study Design}

Six healthy male Japanese white rabbits (JW AIZ) (mean weight, $3.23 \mathrm{~kg}$; range, $3.06-3.55 \mathrm{~kg}$ ) were anesthetized with an injection of sodium pentobarbital (30 $\mathrm{mg} / \mathrm{kg}$ intravenously) through the ear vein and placed in a prone position on a fixation bed. Stannous phytate kits were obtained from Daiichi Radioisotope Laboratories. A ${ }^{99 \mathrm{~m}} \mathrm{Tc}$-phytate solution was prepared by mixing the content of the product and ${ }^{99 \mathrm{~m}} \mathrm{Tc}$-pertechnetate. With a 27 -gauge needle attached to a $1.0-\mathrm{mL}$ syringe, a dose of $18.5 \mathrm{MBq}$ of ${ }^{99 \mathrm{~m}} \mathrm{Tc}$-phytate in $0.2 \mathrm{~mL}$ was injected into 5 sites: the right side of the tongue (submucosally), forelegs (right side subdermally, left side intramuscularly), and posterior legs (right side subdermally, left side intramuscularly). ${ }^{99 m}$ Tc-phytate lymphoscintigraphy of the whole body was performed for all 6 rabbits.

Rabbits were imaged with the CGC at 1,2, 4, and $6 \mathrm{~h}$ after injection with $10 \mathrm{~min}$ of exposure for each whole static image $(512 \times 512$ pixels $)$. Immediately after each CGC imaging study, the detector of the SSGC was placed on the body surface for each of the specified regions, and images were obtained with 5-, 10-, and 15-s acquisition times. Acquisition times for the SSGC were

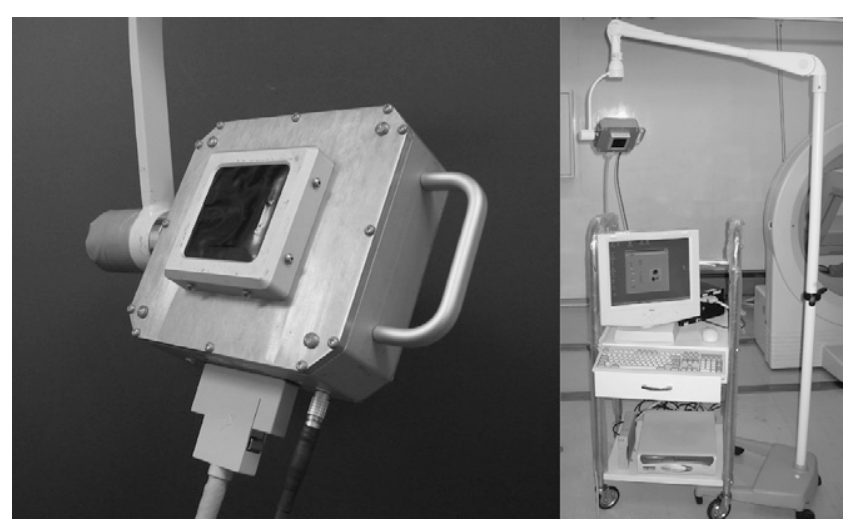

FIGURE 1. SSGC. (Left) SSGC head. (Right) Entire SSGC system. based on previous results (1). The routine acquisition time for the CGC was applied to produce comparable images.

Regions of interest (ROIs) were placed over the hot node and a surrounding background region on CGC-generated static images by use of square templates $(15.2 \times 15.2 \mathrm{~mm})$ to obtain uptake ratios. The radioactivity in each ROI was integrated for $10 \mathrm{~min}$. The isotope uptake was expressed as a ratio of the hot-node activity to the background activity (HN/BG) to compare potential changes in the regional ${ }^{99 \mathrm{~m}} \mathrm{Tc}$-phytate distribution over time.

HN/BG ratios also were determined in SSGC studies with square ROIs of $11 \times 11$ pixels $(15.4 \times 15.4 \mathrm{~mm}$; approximately identical in size to the ROIs in the CGC studies), as in the CGC studies but with a different acquisition time (15 s).

After completion of the last imaging study, rabbits were euthanized by injection of pentobarbital at $100 \mathrm{mg} / \mathrm{kg}$ of body weight or more via the ear vein and dissected to remove tissues containing increased radioactivity in the neck and foreleg regions but not the posterior leg regions. Both SSGC and CGC were used to search for increased radioactivity. The biodistribution of radioactivity in excised specimens was confirmed by autoradiography. The radioactivity in excised tissues was measured with a $\gamma$-well counter. Tissue specimens were fixed in formalin, embedded in paraffin, and stained with hematoxylin-eosin. The study was approved by the Ethics Committee for Animal Research, The Nippon Dental University School of Life Dentistry at Niigata.

Autoradiography was performed by use of a phosphorimager (FLA2000; Fuji Film) with an imaging plate (IP) (BAS-SR2040; Fuji Film). The excised radioactive specimens were weighed, measured in length, and placed on the IP covered with a sheet of film of polyvinylidene. The radioactivity was exposed on the IP for 5 and $10 \mathrm{~min}$ to produce autoradiographic images in a darkroom, and the IP was processed to image the radioactivity by use of the FLA2000 device.

The radioactivities in the specimens and a set of reference ${ }^{99 \mathrm{~m}} \mathrm{Tc}$ radioactivities were assayed with an Autowell $\gamma$-system (ARC-370 M; Aroca). The radioactivities were corrected for decay time, calculated, and expressed as percentages (activity in the specimen relative to the initial injected radioactivity).

Data on percentage variability were expressed as mean \pm SD. A 2-tailed paired Student $t$ test was used for 2-group comparisons for SSGC versus CGC. Probability values of less than 0.05 were considered statistically significant.

\section{Clinical Study Design}

Eight patients ( 4 men and 4 women; age [mean \pm SD], $53.3 \pm$ 21.0 y) with oral cancer were enrolled in the clinical study at Niigata Hospital (The Nippon Dental University School of Life Dentistry at Niigata). Primary lesions were in the tongue $(n=7)$ and lower gingiva $(n=1)$, and the stages were N0 $(n=3)$, N1 $(n=2)$, and N2c $(n=3)$. Sentinel node lymphoscintigraphy was performed after the injection of ${ }^{99 \mathrm{~m}} \mathrm{Tc}$-phytate (Daiichi Radioisotope Laboratories). The radiopharmaceutical was injected into 4 healthy sites surrounding the tumor and approximately $5 \mathrm{~mm}$ from the edge of the tumor $(18.5 \mathrm{MBq} / 0.25 \mathrm{~mL} \times 4)$ with a 27 -gauge needle after the application of topical anesthetic. The injection involved creating a vesicle to attain administration to a submucosal depth. Although an N2c neck lesion was not an indication for sentinel lymph node biopsy, the 3 patients who had such lesions were included in the study simply for lymphoscintigraphy.

Sentinel lymph node biopsy had not been introduced to the hospital at the time of this clinical study. Informed consent was 
obtained from the patients after explanation of the nature and possible risks of their voluntary participation in the clinical study. This research was approved by The Ethics Committee, The Nippon Dental University School of Life Dentistry at Niigata.

The same SSGC as that used in the animal study was applied in the clinical study. First, comparative CGC images were obtained at 1, 2, 4, 6, and $24 \mathrm{~h}$ after injection. SSGC studies followed CGC studies at each time setting, and images were obtained with 5-, 10-, and 15 -s acquisition times. Occasionally, additional 30- or 60-s images were obtained. Comparable CGC images were obtained with routine 10-min acquisition times. Head and neck imaging with CGC was performed with patients in the prone position. Posterior-to-anterior and lateral projection images were completed with 10-min acquisition times. SPECT also was performed at $4 \mathrm{~h}$ after injection. ROIs were placed over the hot node and the surrounding background area on both SSGC and CGC static images, as in the animal study, to obtain HN/BG ratios. The lymphoscintigrams obtained with both the SSGC and the CGC were interpreted by 3 independent radiologists who were experts in the field of body imaging. The uptake of ${ }^{99 \mathrm{~m}} \mathrm{Tc}$-phytate was evaluated subjectively by consensus of the radiologists.

The HN/BG ratios were expressed as mean $\pm \mathrm{SD}$ and compared. An ANOVA was used to compare the ratios for the SSGC and the CGC. Probability values of less than 0.05 were considered statistically significant.

\section{RESULTS}

\section{Animal Study}

In the animal study, the SSGC detected a total of 29 hot nodes in images of 6 rabbits, each at 2, 4, and $6 \mathrm{~h}$ after injection (15-s acquisition time). The number of hot nodes detected was the same as the number detected by the CGC: 11 in the neck region, 6 in the left anterior leg region, 7 in the right anterior leg region, 2 in the left posterior leg region, and 3 in the right posterior leg region. The SSGC clearly depicted the increased radioactivity at shorter acquisition times $(5,10$, and $15 \mathrm{~s})$ than the CGC (standard 600-s acquisition time) (Fig. 2).

The HN/BG ratios of 2-h images (9 nodes) and 4-h images (11 nodes) of 6 rabbits were calculated in both $\gamma$-camera studies. The HN/BG ratios for the SSGC were $38.1 \pm 32.9(2 \mathrm{~h})$ and $39.3 \pm 30.5(4 \mathrm{~h})$. The HN/BG ratios for the CGC were $31.6 \pm 35.4(2 \mathrm{~h})$ and $29.4 \pm 26.6(4 \mathrm{~h})$. There were no significant differences in the HN/BG ratios between the $\gamma$-cameras $(P=0.864)$ (Fig. 3).

All 22 tissues in the neck and anterior leg regions with increased radioactivities were excised and confirmed by microscopic examination to be lymph nodes. An autoradiographic study showed that all 22 lymph nodes were radioactive. The weights (mean \pm SD) of the lymph nodes were $0.14 \pm 0.09 \mathrm{~g}$ in the neck, $0.05 \pm 0.07 \mathrm{~g}$ in the anterior leg regions, and $0.09 \pm 0.08 \mathrm{~g}$ for all 22 lymph nodes. The mean maximum lengths of the lymph nodes were $12.9 \pm$ $6.4 \mathrm{~mm}$ in the neck, $5.5 \pm 3.8 \mathrm{~mm}$ in the anterior leg regions, and $8.9 \pm 6.0 \mathrm{~mm}$ for all 22 lymph nodes.

Radioactivity in the excised specimens was assayed with a $\gamma$-well counter. The mean percentages of radioactivity in the excised lymph nodes relative to the initial injected

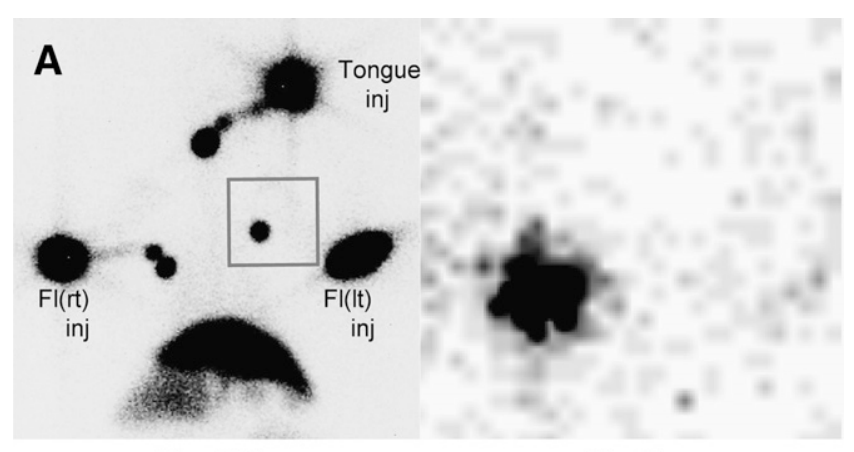

$4 \mathrm{~h}, 600 \mathrm{~s}$

$4 \mathrm{~h}, 5 \mathrm{~s}$

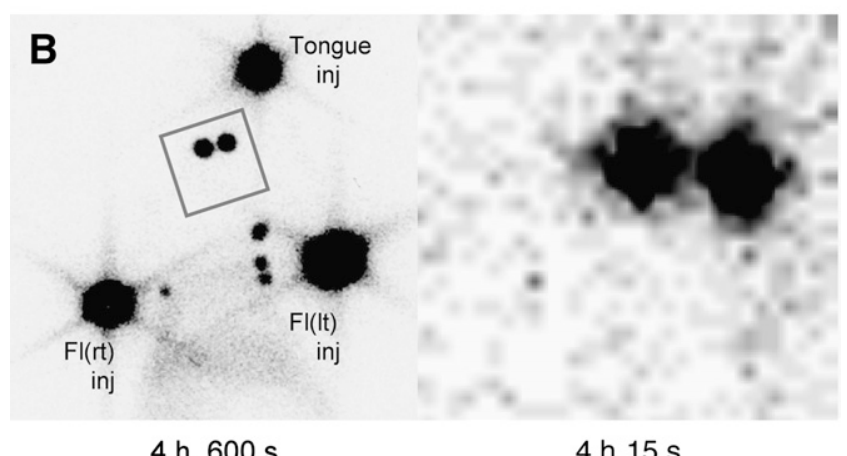

$4 \mathrm{~h}, 600 \mathrm{~s}$

$4 \mathrm{~h}, 15 \mathrm{~s}$

FIGURE 2. Comparison of lymphoscintigrams of rabbits. (A) (Left) CGC image shows hot nodes at $4 \mathrm{~h}$ after injection with acquisition time of $600 \mathrm{~s}$. Hot node in left foreleg region is circumscribed by rectangular frame. (Right) SSGC image at $4 \mathrm{~h}$ after injection with 5-s acquisition time shows same hot node. (B) (Left) Two sentinel nodes in neck region are shown in rectangular frame on CGC image after 600-s acquisition time. (Right) These 2 sentinel nodes are shown on SSGC image with 15-s acquisition time ( $4 \mathrm{~h}$ after injection). $\mathrm{FI}=$ foreleg; inj = injection site; It = left; $r t=$ right.

radioactivity were $0.21 \% \pm 0.18 \%$ for lymph nodes in the neck, $0.12 \% \pm 0.11 \%$ for lymph nodes in the anterior leg regions, and $0.15 \% \pm 0.15 \%$ for all 22 lymph nodes. The percentages ranged from $0.01 \%$ to $0.62 \%$ (Fig. 4 and Table 1). The 3 lymph nodes containing $0.01 \%$ of the initial injection volume (18.5 MBq of ${ }^{99 \mathrm{~m}} \mathrm{Tc}$ ) were detected by both $\gamma$-cameras at $6 \mathrm{~h}$ after injection.

\section{Clinical Study}

The SSGC detected all hot nodes that were detected by the CGC in 8 patients with oral cancer (10 neck sites). The numbers of hot nodes in the neck were $31,29,30,27$, and 16 at 1, 2, 4, 6, and $24 \mathrm{~h}$ after injection, respectively (Table $2)$. The hot nodes were revealed in the following regions at $4 \mathrm{~h}$ after injection: 7 hot nodes at level I, 18 hot nodes at level II, 3 hot nodes at level III, and 2 hot nodes at level IV. The node-level classification is from the American Joint Committee on Cancer (2002). Eighteen of the 30 hot nodes $(60 \%)$ were localized at level II. The SSGC revealed the hot nodes with short acquisition times (Fig. 5).

Eighteen hot nodes from 8 patients were chosen for determining the HN/BG ratios for the SSGC and the CGC 


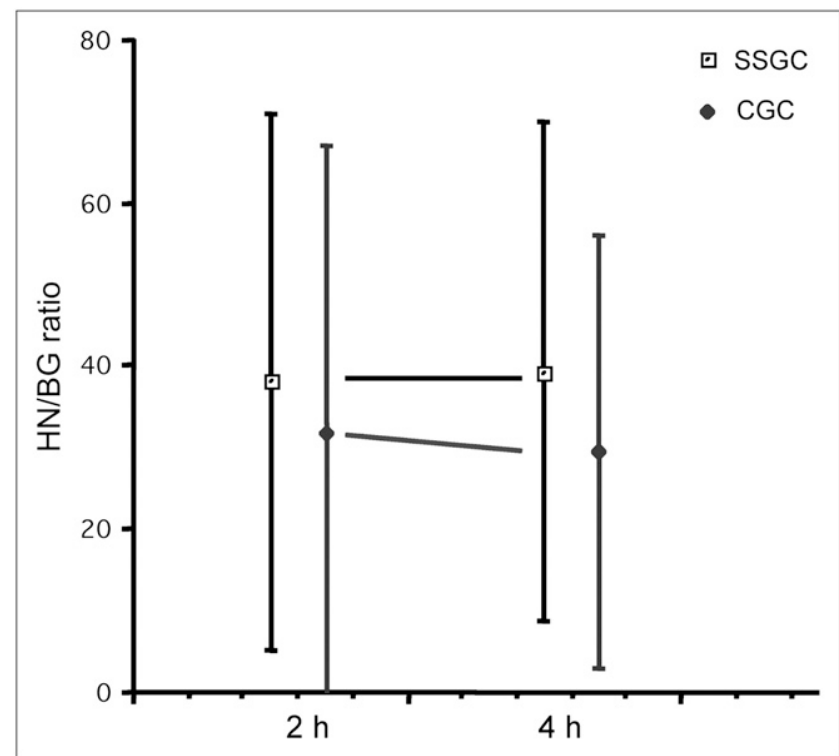

FIGURE 3. Comparison of HN/BG ratios between SSGC and CGC in animal study (9 hot nodes at $2 \mathrm{~h} ; 11$ hot nodes at $4 \mathrm{~h}$ ), demonstrating no significant differences $(P=0.864)$.

to compare image quality. The mean HN/BG ratios of the 18 hot nodes for the SSGC were $10.9 \pm 7.1,9.6 \pm 6.3$, and $4.3 \pm 1.7$ at 2,4 , and $24 \mathrm{~h}$ after injection, respectively. The mean HN/BG ratios of the hot nodes for the CGC were $8.8 \pm 5.6,7.1 \pm 4.4$, and $3.1 \pm 1.7$ at 2,4 , and $24 \mathrm{~h}$ after
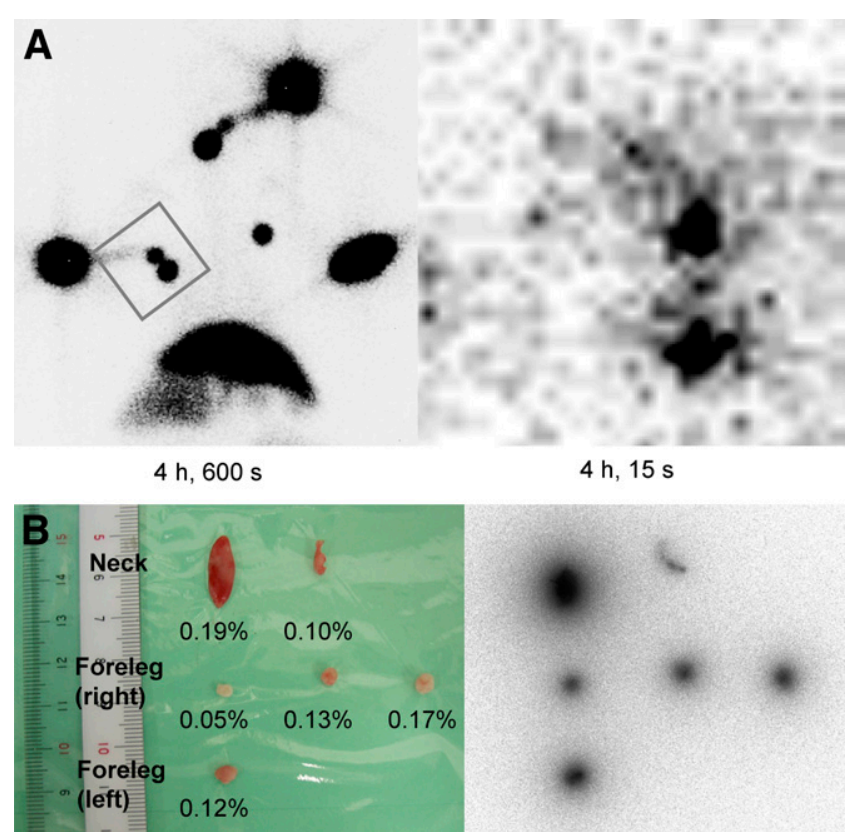

FIGURE 4. Confirmation of radioactivity in excised specimens by autoradiography. (A) (Left) CGC image at $4 \mathrm{~h}$ after injection with 600-s acquisition time. (Right) SSGC image at $4 \mathrm{~h}$ after injection with 15-s acquisition time. (B) (Left) Three specimens excised from right foreleg region contained high levels of radioactivity (percentage of initial injection of ${ }^{99 m T c}$-phytate). (Right) Autoradiographic image of specimens. injection, respectively. There were no significant differences in the HN/BG ratios between the $\gamma$-cameras $(P=$ 0.969) (Fig. 6).

\section{DISCUSSION}

Sentinel lymph node biopsy is an established and widely available procedure for breast cancer and melanoma (1625). It is also used for the management of head and neck cancer and other malignancies. Various ${ }^{99 \mathrm{~m}} \mathrm{Tc}$ radiocolloids are used for this technique. In breast cancer, the doses of injected radiopharmaceuticals have been reported to be 5-10 MBq (18), 10-20 MBq (19), and 3.7-111 MBq (20). Similar doses (15-111 MBq) have been used for the procedure in patients who have oral cancer (21-25).

When a sentinel lymph node biopsy is performed, only a very small amount of radiopharmaceutical accumulates in the sentinel node or nodes. Although the injection methods and ${ }^{99 \mathrm{~m}} \mathrm{Tc}$-labeled particles differed, the average accumulated radioactivities relative to the injected doses per lymph node in breast cancer studies were reported to be $0.9 \%$ and $0.60 \%(26,27)$, and the range was $0.001 \%-2.5 \%(27,28)$. Some surgeons have estimated the percentage of the injected dose per lymph node to be about $0.15 \%$.

It is a challenge to visualize the small amount of radioactivity in the target tissue by using a small $\gamma$-camera during the procedure. In our previous simulation study, the SSGC reliably detected small amounts of radioactivity (150 $\mathrm{kBq} ; 0.15 \%$ of an estimated injected dose of $100 \mathrm{MBq}$ of ${ }^{99 \mathrm{~m}} \mathrm{Tc}$ ) in simulated nodes in a water bath at up to a 30-mm underwater depth after $18 \mathrm{~h}$. Even after $24 \mathrm{~h}$, when the radioactivity had decreased to $1 / 16$ th of its initial value (9.4 $\mathrm{kBq}$ ), the simulated sentinel nodes still could be recognized with short acquisition times (1).

In the animal study, the SSGC clearly depicted sentinel lymph nodes after ${ }^{99 m}$ Tc-phytate injection. The percentage of injected radioactivity (18.5 MBq) in hot nodes ranged from $0.01 \%$ to $0.62 \%$, with an average of $0.15 \%$ at $6 \mathrm{~h}$ after the injection. At the time of the last imaging study, the initial radioactivity had decreased by almost $50 \%$ with the physical decay of ${ }^{99 \mathrm{~m}} \mathrm{Tc}$, indicating that the SSGC could image the radioactivity of at least $0.00093 \mathrm{MBq}$ in a hot node. The SSGC revealed all hot nodes that were revealed by the CGC, and the acquisition times of the SSGC were very short $(5,10$, or $15 \mathrm{~s})$ compared with the usual 5 or 10 min required for the CGC. The absence of radioactivity in the lymphatic basin was confirmed easily by the SSGC after the excision of a hot node or nodes, especially close to the injection site. Furthermore, the SSGC provided simple verification of radioactivity in specimens after surgery.

The clinical study also showed the favorable performance of the SSGC in detecting hot nodes. The SSGC detected all hot nodes detected by the CGC, but with short acquisition times $(5,10,15,30$, and $60 \mathrm{~s})$. In both animal and clinical studies, sentinel lymph nodes, even lymphatic channels in some images, were clearly shown by the SSGC. 
TABLE 1

Lymph Node Weight and Percentage of Dose ( $\left.{ }^{99 m} \mathrm{Tc}\right)$ in Nodes

\begin{tabular}{|c|c|c|c|c|}
\hline \multirow[b]{2}{*}{ Rabbit } & \multicolumn{2}{|c|}{ Neck region (10 hot nodes) } & \multicolumn{2}{|c|}{ Foreleg region (12 hot nodes) } \\
\hline & Lymph node weight (g) & $\%$ of dose $\left({ }^{99 m T c}\right)$ in node & Lymph node weight (g) & $\%$ of dose $\left({ }^{99 m T c)}\right.$ in node \\
\hline \multirow[t]{2}{*}{1} & 0.22 & 0.14 & 0.18 & 0.30 (right) \\
\hline & & & 0.08 & 0.34 (left) \\
\hline \multirow[t]{2}{*}{2} & 0.27 & 0.62 & & \\
\hline & 0.04 & 0.01 & & \\
\hline \multirow[t]{2}{*}{3} & 0.13 & 0.33 & 0.01 & 0.06 (right) \\
\hline & 0.01 & 0.33 & & \\
\hline \multirow[t]{4}{*}{4} & 0.20 & 0.08 & 0.03 & 0.01 (right) \\
\hline & 0.14 & 0.08 & 0.08 & 0.05 (left) \\
\hline & & & 0.01 & 0.01 (left) \\
\hline & & & 0.02 & 0.02 (left) \\
\hline \multirow[t]{4}{*}{5} & 0.15 & 0.19 & 0.03 & 0.17 (right) \\
\hline & 0.02 & 0.10 & 0.02 & 0.13 (right) \\
\hline & & & 0.01 & 0.05 (right) \\
\hline & & & 0.03 & 0.12 (left) \\
\hline 6 & 0.19 & 0.21 & 0.09 & 0.39 (left) \\
\hline
\end{tabular}

The SSGC could reveal more details than the CGC at shorter acquisition times. In the previous simulation study, we tested the SSGC with the "shine through" phenomenon, in which too much radioactivity from the injection site interferes with the detection of a small amount of nodal radioactivity situated close to the injection site during the exploration of sentinel lymph nodes with a $\gamma$-probe. The SSGC could resolve a simulated sentinel lymph node situated close to the injection site. In both of the present studies, the SSGC demonstrated lymph nodes neighboring the injection site.

Real-time imaging also was attained in the clinical experiment; such imaging may provide surgeons with increased maneuverability for surgical procedures.

The favorable results obtained in our animal and clinical studies were primarily attributable to the excellent basic performance of the SSGC, described earlier in this article. The basic performance seems to be notable, even among small $\gamma$-cameras with scintillation-based technology $(8-12)$ or semiconductor technology $(13,14)$.

The camera head for handheld small $\gamma$-cameras weighs as little as $820 \mathrm{~g}-2.7 \mathrm{~kg}(1,10,11,13-15)$. The field of view of such cameras varies: $20 \times 20 \mathrm{~mm}(9), 24 \mathrm{~mm}$ in diameter (12), $32 \times 32 \mathrm{~mm}(13), 40 \mathrm{~mm}$ in diameter (10), $40 \times$ $40 \mathrm{~mm}(14), 44 \times 44 \mathrm{~mm}(15), 44.8 \times 44.8 \mathrm{~mm}(1,15)$, and $95 \times 95 \mathrm{~mm}$ (15). However, these handheld small $\gamma$-cameras may not replace $\gamma$-probes completely in radioguided surgery because their detector heads still obscure the surgical field. The SSGC does offer a tremendous advantage with visualization of the target uptake during surgery. Furthermore, surgeons can use the SSGC to confirm the location of radioactivity in excised specimens during surgery. Recently, the usefulness of a handheld miniature $\gamma$-camera for parathyroidectomy was reported (11). In a commentary about the article, Rubello and Mariani speculated that the combined use of a $\gamma$-probe and the handheld miniature

TABLE 2

Number of Nodes and HN/BG Ratios for Nodes with Increased Radioactivity Identified by SSGC and CGC at 4 Hours After Injection

\begin{tabular}{ccclcll}
\hline Patient & Age $(\mathrm{y})$ & Sex & $\begin{array}{c}\text { Primary } \\
\text { region }\end{array}$ & TNM & $\begin{array}{c}\text { No. of hot nodes } \\
\left.\text { identified by SSGC (HN/BG ratio }{ }^{\dagger}\right)\end{array}$ & $\begin{array}{c}\text { No. of hot nodes } \\
\left.\text { identified by CGC (HN/BG ratio }{ }^{\dagger}\right)\end{array}$ \\
\hline 1 & 66 & F & Tongue & T1N0M0 & $6(12.8,23.3,6.9)$ & $6(6.6,12.9,2.9)$ \\
2 & 26 & M & Tongue & T2N0M0 & $3(24.5,8.2)$ & $3(18.0,5.2)$ \\
3 & 42 & F & Tongue & T2N0M0 & $2(13.2,12.1)$ & $2(13.0,11.4)$ \\
4 & 34 & M & Tongue & T1N1M0 & $3(12.4,8.6,4.0)$ & $3(9.4,8.2,3.5)$ \\
5 & 58 & M & Tongue & T2N1M0 & $5(4.9,6.6,4.3)$ & $5(4.1,5.4,3.7)$ \\
6 & 45 & F & Tongue & T3N2cM0 & $3(4.4)$ & $3(3.1)$ \\
7 & 63 & M & Tongue & T3N2cM0 & $6(7.9,7.3,7.3)$ & $6(7.9,5.6,4.7)$ \\
8 & 92 & F & Lower gingiva & T4N2cM0 & $2(2.4)$ & $2(1.6)$
\end{tabular}

${ }^{*}$ All lesions were squamous cell carcinomas.

${ }^{\dagger} \mathrm{HN} / \mathrm{BG}$ ratios of 18 hot nodes are shown. 

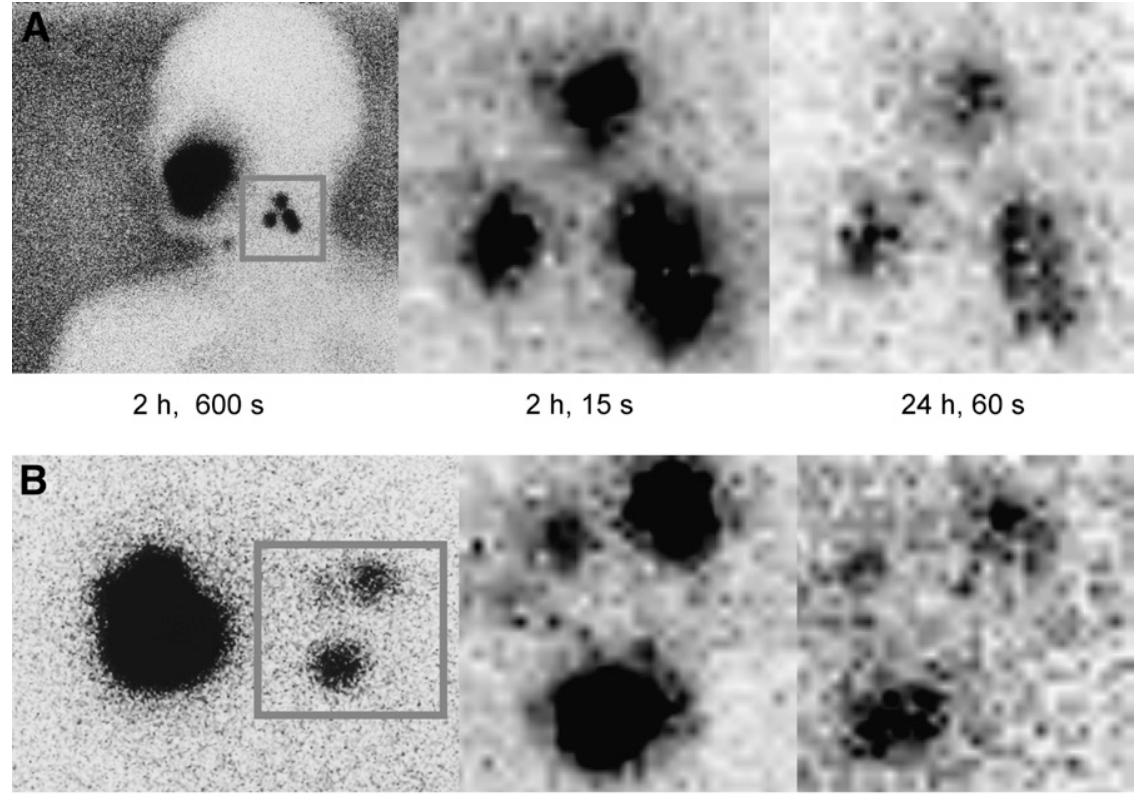

$24 \mathrm{~h}, 600 \mathrm{~s}$
$24 \mathrm{~h}, 30 \mathrm{~s}$
FIGURE 5. (A) T2N1M0 (pN0) squamous cell carcinoma of tongue in 58-yold man. (Left) CGC image shows neck hot nodes (2 h after injection, 600-s acquisition time). (Middle) SSGC image clearly shows 4 hot nodes with short (15s) acquisition time. (Right) Sentinel nodes are shown with acquisition time of $60 \mathrm{~s}$ at $24 \mathrm{~h}$ after injection. (B) T1N1M0 (pN0) squamous cell carcinoma of tongue in 34-y-old man. (Left) CGC image shows neck hot nodes (24 h after injection, $600-\mathrm{s}$ acquisition time). (Middle) SSGC image clearly shows lymphatic vessel at $6 \mathrm{~h}$ after injection with acquisition time of $15 \mathrm{~s}$. (Right) Three hot nodes are shown at $24 \mathrm{~h}$ after injection (acquisition time, $30 \mathrm{~s}$ ). $\gamma$-camera would be practical for surgery, especially when the target tissues are located ectopically or deeply (29). We agree with that approach for sentinel lymph node biopsy; the simultaneous use of the devices better facilitated the exploration of hot nodes than did the independent use of each device in the animal study.

We have reported the performance of an experimental type of clinical SSGC that has been modified for better maneuverability during surgery. The size of the detector head of this experimental SSGC was greatly decreased by the architectural design of the configuration of the detector. The weight of the device was minimized to $1.4 \mathrm{~kg}$ (Fig. 7) $(30,31)$.

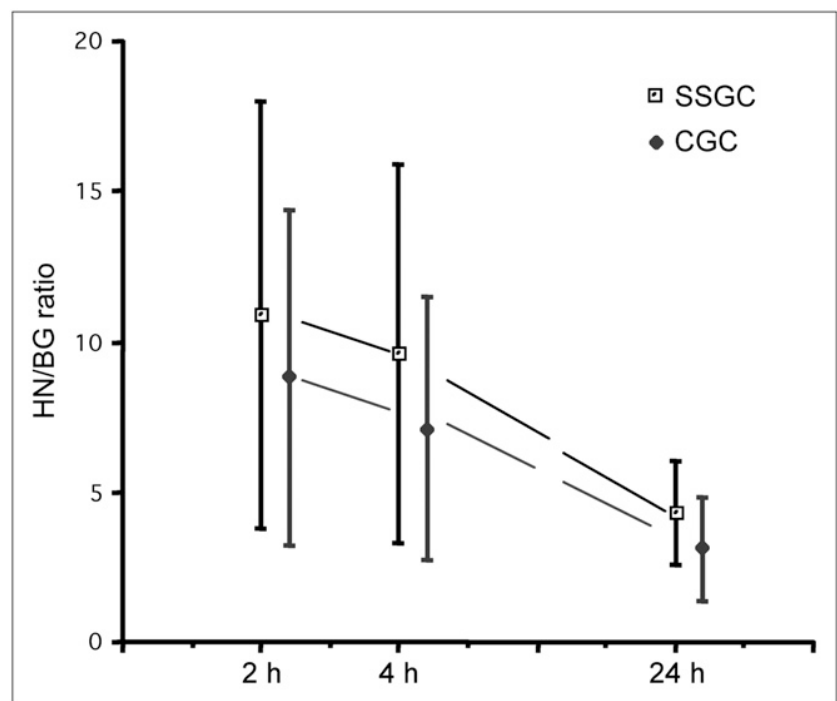

FIGURE 6. Comparison of HN/BG ratios between SSGC and CGC in clinical study $(n=18)$, demonstrating no significant differences $(P=0.969)$.

\section{CONCLUSION}

A prototype SSGC for surgical use was able to resolve the small amount of radioactivity in sentinel nodes in an animal study of sentinel lymph node biopsy. The mean radioactivity in imaged nodes was $0.15 \%$ of the initial injected volume of ${ }^{99 m} \mathrm{Tc}$-phytate $(18.5 \mathrm{MBq})$. Furthermore, the SSGC clearly depicted increased uptake in the necks of patients with oral cancer at shorter acquisition times than did the CGC. These depictions were confirmed by a semiquantitative method (determination of HN/BG ratios) in both animal and clinical studies. We verified the efficacy of the SSGC for detecting a small amount of radioactivity in hot nodes during the procedure. The SSGC can offer clear visualization of sentinel nodes during surgery.

\section{ACKNOWLEDGMENT}

This study was supported in part by Grants-in-Aid for Scientific Research from the Ministry of Education, Science, Sports and Culture of Japan (B2-11557142 and B1-14370682).

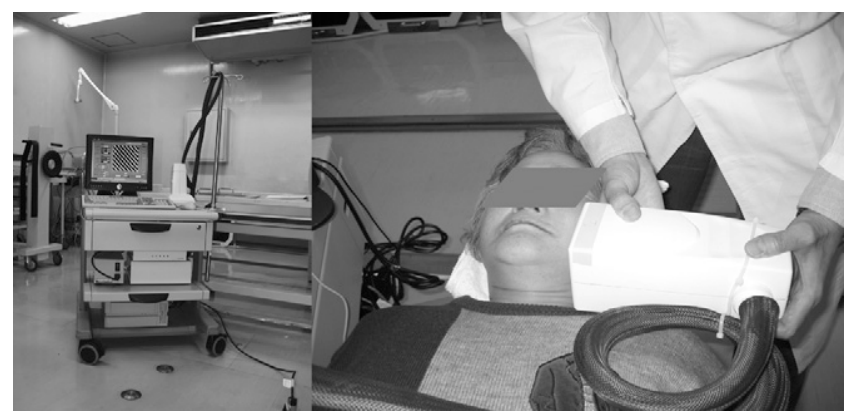

FIGURE 7. SSGC designed for clinical use. 


\section{REFERENCES}

1. Tsuchimochi M, Sakahara H, Hayama K, et al. A prototype small CdTe gamma camera for radioguided surgery and other imaging applications. Eur J Nucl Med Mol Imaging. 2003;30:1605-1614.

2. Entine G, Luthmann R, Mauderli W, Fitzgerald LT, Williams CM. Cadmium telluride gamma camera. IEEE Trans Nucl Sci. 1979;NS-26:552-558.

3. Matherson KJ, Barber HB, Barrett HH, et al. Progress in the development of large-area modular $64 \times 64 \mathrm{CdZnTe}$ imaging arrays for nuclear medicine. IEEE Trans Nucl Sci. 1988;45:354-358.

4. Funaki M, Ozaki T, Satoh K, Ohno R. Growth and characterization of CdTe single crystals for radiation detectors. Nucl Instrum Methods. 1999;A436:120-126.

5. Hoffman EJ, Tornai MP, Janecek M, Patt BE, Iwanczyk JS. Intraoperative probes and imaging probes. Eur J Nucl Med. 1999;26:913-935.

6. Mori I, Takayama T, Motomura N. The CdTe detector and its imaging performance. Ann Nucl Med. 2001;15:487-494.

7. Mueller B, O'Connor MK, Blevis I, et al. Evaluation of a small cadmium zinc telluride detector for scintimammography. J Nucl Med. 2003;44:602-609.

8. Patt BE, Tornai MP, Iwanczyk JS, Levin CS, Hoffman EJ. Development of an intraoperative gamma camera based on a 256-pixel mercuric iodine detector array. IEEE Trans Nucl Sci. 1997;44:1242-1248.

9. MacDonald LR, Patt BE, Iwanczyk JS, et al. High-resolution hand-held gamma camera. In: Barber HB, Roehrig H, Morton EJ, eds. Penetrating Radiation Systems and Applications II: 2-3 August 2000, San Diego, CA. Bellingham, WA: SPIE; 2000:242-253.

10. Pitre S, Menard L, Ricard M, et al. A hand-held imaging probe for radio-guided surgery: physical performance and preliminary clinical experience. Eur J Nucl Med Mol Imaging. 2003;30:339-343.

11. Ortega J, Ferrer-Rebolleda J, Cassinello N, Lledo S. Potential role of a new hand-held miniature gamma camera in performing minimally invasive parathyroidectomy. Eur J Nucl Med Mol Imaging. 2007;34:165-169.

12. Menard L, Charon Y, Solal M, et al. A compact high resolution gamma camera for intra-operative surgical use. IEEE Trans Nucl Sci. 1998;45:1293-1297.

13. Abe A, Takahashi N, Lee J, et al. Performance evaluation of a hand-held, semiconductor (CdZnTe)-based gamma camera. Eur J Nucl Med Mol Imaging. 2003;30:805-811.

14. Kopelman D, Blevis I, Iosilevsky G, et al. A newly developed intra-operative gamma camera: performance characteristics in a laboratory phantom study. Eur J Nucl Med Mol Imaging. 2005;32:1217-1224.

15. Sánchez F, Fernández MM, Giménez M, et al. Performance tests of two portable mini gamma cameras for medical applications. Med Phys. 2006;33:4210-4220.

16. Keshtgar MR, Ell PJ. Sentinel lymph node detection and imaging. Eur J Nucl Med. 1999;26:57-67.

17. Morton DL, Thompson JF, Essner R, et al. Validation of the accuracy of intraoperative lymphatic mapping and sentinel lymphadenectomy for early-stage melanoma: a multicenter trial. Multicenter Selective Lymphadenectomy Trial Group. Ann Surg. 1999;230:453-463.

18. Veronesi U, De Palo G, Marubini E, et al. Randomized trial of fenretinide to prevent second breast malignancy in women with early breast cancer. $J$ Natl Cancer Inst. 1999;91:1847-1856.

19. Mariani G, Moresco L, Viale G, et al. Radioguided sentinel lymph node biopsy in breast cancer surgery. $J$ Nucl Med. 2001;42:1198-1215.

20. Schwartz GF, Giuliano AE, Veronesi U; Consensus Conference Committee. Proceedings of the consensus conference on the role of sentinel lymph node biopsy in carcinoma of the breast, April 19-22, 2001, Philadelphia, Pennsylvania. Cancer. 2002;94:2542-2551.

21. Shoaib T, Soutar DS, Prosser JE, et al. A suggested method for sentinel node biopsy in squamous cell carcinoma of the head and neck. Head Neck. 1999;21: 728-733.

22. Klutmann S, Bohuslavizki KH, Brenner W, et al. Lymphoscintigraphy in tumors of the head and neck using double tracer technique. J Nucl Med. 1999;40:776-782.

23. Taylor RJ, Wahl RL, Sharma PK, et al. Sentinel node localization in oral cavity and oropharynx squamous cell cancer. Arch Otolaryngol Head Neck Surg. 2001;127:970-974.

24. Ross G, Shoaib T, Soutar DS, et al. The use of sentinel node biopsy to upstage the clinically N0 neck in head and neck cancer. Arch Otolaryngol Head Neck Surg. 2002;128:1287-1291.

25. Ross GL, Shoaib T. Role of sentinel node biopsy in the management and staging of the N0 neck. Odontology. 2005;93:1-6.

26. De Cicco C, Cremonesi M, Luini A, et al. Lymphoscintigraphy and radio-guided biopsy of the sentinel axillary node in breast cancer. J Nucl Med. 1998;39:20802084.

27. Rink T, Heuser T, Fitz H, Schroth H-J, Weller E, Zippel HH. Lymphoscintigraphic sentinel node imaging and gamma probe detection in breast cancer with ${ }^{99 \mathrm{~m}} \mathrm{Tc}$ nanocolloidal albumin: results of an optimized protocol. Clin Nucl Med. 2001;26:293-298.

28. Nieweg OE, Tanis PJ, de Vries JDH, Valdes Olmos RA, Kroon BBR, Hoefnagel CA. The sentinel node in melanoma: present controversies. Handout book presented at: 48th Society of Nuclear Medicine Annual Meeting; Toronto, Canada; June 26, 2001:94-103.

29. Rubello D, Mariani G. Hand-held gamma probe or hand-held miniature gamma camera for minimally invasive parathyroidectomy: competition, evolution or synergy? Eur J Nucl Med Mol Imaging. 2007;34:162-164.

30. Hayama K, Tsuchimochi M, Sakahara H, Oda T. The basic performance of an experimental type of clinical small semiconductor gamma camera [abstract; in Japanese]. Kaku Igaku. 2005;42:350.

31. Tanaka C, Fujii H, Shiotani A, Kitagawa Y, Nakamura K, Kubo A. Sentinel node imaging of laryngeal cancer using a portable gamma camera with CdTe semiconductor detectors. Clin Nucl Med. 2005;30:440-443. 